Vol. 86 No. 2 June 2021

\title{
CYTOLOGIA
}

International Journal of Cytogenetics and Cell Biology 


\title{
Technical Note:
}

\section{Environmental Responses of the Membrane Trafficking Factor PATROL1 in the Arabidopsis Stomatal Complex}

\author{
Takumi Higaki $^{1 *}$, Fumiya Sato ${ }^{2}$ and Koh Iba ${ }^{3}$ \\ ${ }^{1}$ International Research Organization for Advanced Science and Technology, Kumamoto University, \\ Kurokami, Chuo-ku, Kumamoto 860-8555, Japan \\ ${ }^{2}$ Graduate School of Science and Technology, Kumamoto University, \\ Kurokami, Chuo-ku, Kumamoto 860-8555, Japan \\ ${ }^{3}$ Department of Biology, Faculty of Science, Kyushu University, Fukuoka 819-0395, Japan
}

Received March 10, 2021; accepted April 2, 2021

The plant stomatal complex regulates the aperture of the stomatal pore in response to changes in environmental factors such as light, water, salinity, and atmospheric gasses. Stomatal opening is achieved by activating a plasma membrane proton pump ATPase $\left(\mathrm{H}^{+}\right.$-ATPase $)$ to induce plasma membrane hyperpolarization, which facilitates potassium influx to guard cells. A membrane trafficking factor named $\mathrm{H}^{+}$-ATPase translocation control 1 (PATROL1) was isolated as a key regulator of stomatal opening in Arabidopsis thaliana under low $\mathrm{CO}_{2}$ conditions (Hashimoto-Sugimoto et al. 2013). PATROL1 has a MUN domain with synaptic vesicle priming activity, which is crucial for neurotransmitter release in animals. The patroll mutation impairs both the plasma membrane localization of the $\mathrm{H}^{+}$-ATPase AHA1 and stomatal opening, suggesting that PATROL1 delivers AHA1 to plasma membranes depending on environmental conditions (Hashimoto-Sugimoto et al. 2013). Confocal imaging revealed that green fluorescent protein (GFP)-tagged PATROL1 (GFP-PATROL1) localized to the cytoplasm and dot-like components on plasma membranes, and the GFP-PATROL1 dots appeared and remained at the same site for $2-10 \mathrm{~s}$, implying exocytosis or endocytosis of plasma membrane components such as AHA1 (Higaki et al. 2014, Higaki 2015).

The cover shows a confocal image of GFP-PATROL1 and red fluorescent protein (RFP)-tagged AHA1 in the stomatal complex of an A. thaliana cotyledon. The cotyledon was detached from a 7-day-old transgenic seedling, and then mounted on a slide glass with a cover glass and a drop of buffer to avoid bubbles between the cotyledon and cover glass (Higaki 2015). The specimen was observed under a fluorescence microscope equipped with confocal spinning disk confocal laser scanning unit (CSU-W1; Yokogawa, Tokyo, Japan) and a complemen- tary metal oxide semiconductor camera (Zyla; Andor, Belfast, UK). GFP and RFP were excited at $488 \mathrm{~nm}$ and $561 \mathrm{~nm}$, respectively, with emission wavelengths of $510-550 \mathrm{~nm}$ and $624-640 \mathrm{~nm}$, respectively.

Our live cell imaging revealed that the GFPPATROL1 dot density reversibly changes in response to environmental conditions; the density in the guard cells increases when the stomata close and decreases when the stomata open (Hashimoto-Sugimoto et al. 2013, Higaki et al. 2014). In addition, we recently found that salinity stress diminished GFP-PATROL1 dots but induced unusual GFP-PATROL1-labeled large bodies and rod-like structures (Sato et al. 2021). Cells under the hyperosmotic condition had similar large bodies, but not rods, indicating that the rod-like GFP-PATROL1-labeled structures form specifically under salinity stress conditions (Sato et al. 2021). In addition, the primary root growth of the patroll mutant was sensitive to salinity stress, suggesting that PATROL1 also contributes to salinity stress tolerance (Sato et al. 2021).

\section{References}

Hashimoto-Sugimoto, M., Higaki, T., Yaeno, T., Nagami, A., Irie, M., Fujimi, M., Miyamoto, M., Akita, K., Negi, J., Shirasu, K., Hasezawa, S. and Iba, K. 2013. A Munc13-like protein in Arabidopsis mediates $\mathrm{H}^{+}$-ATPase translocation that is essential for stomatal responses. Nat. Commun. 4: 1-9.

Higaki, T. 2015. Real-time imaging of plant cell surface dynamics with variable-angle epifluorescence microscopy. J. Vis. Exp. 106: e53437.

Higaki, T., Hashimoto-Sugimoto, M., Akita, K., Iba, K. and Hasezawa, S. 2014. Dynamics and environmental responses of PATROL1 in Arabidopsis subsidiary cells. Plant Cell Physiol. 55: 773-780.

Sato, F., Iba, K. and Higaki, T. 2021. Involvement of the membrane trafficking factor PATROL1 in the salinity stress tolerance of Arabidopsis thaliana. Cytologia 86: 119-126.

*Corresponding author, e-mail: thigaki@kumamoto-u.ac.jp

DOI: $10.1508 /$ cytologia.86.101 